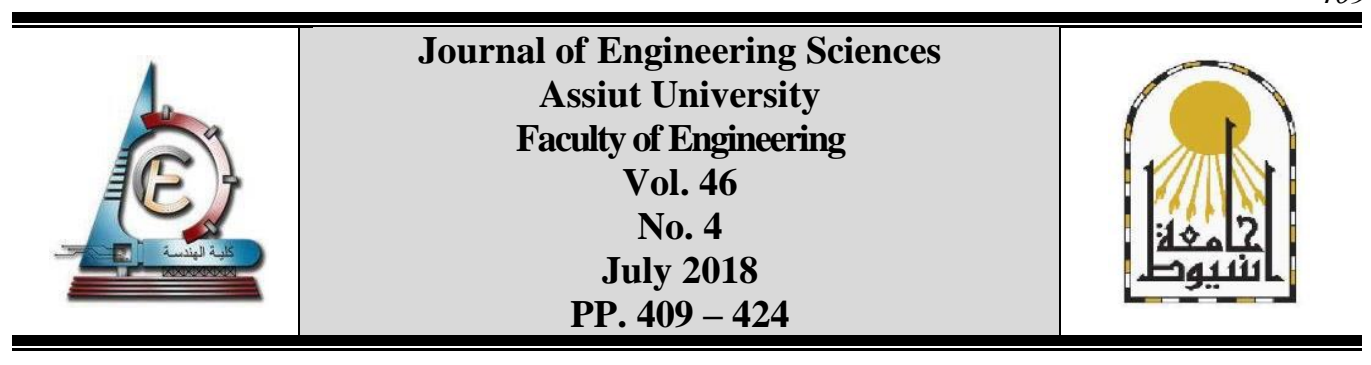

\title{
PROFIT DISTRIBUTION IN MICRO-GRID
}

\author{
Hala G. Farrag ", Reham S.Saad, Mostafa M. Fouda, and Hala A. K. Mansour \\ Electrical Eng. Deart., Faculty of Engineering at Shoubra, Benha University, Egypt.
}

Received 19 March 2018; Accepted 21 April 2018

\begin{abstract}
Smart-grids are advanced power systems that have capability to control the power loading by saving the purchasing cost of extra electricity and alleviating power losses. The communication between the micro station (MS) and Micro-grid (MGs) is a key issue in smart grid where its performance affected by transmission pricing and extra exchanged power pricing that considers an efficient role on the pricing stability of the smart micro-grids. Therefore, interchange mechanism is proposed in (MGs) to integration, fulfilment of all requirements and enhanced routing of communication in smart-grids. We study the power consumption and the load-price mapping for coalitions with lower repetition time and equal contribution to fulfil all requirements. This achieved by means of optimization problem that increment of total revenue, which based on the optimization tool function. We used Coalition Game Theoretical Formulation Strategy (CG-TFS) to meet the high-speed routing of smart grid requirements and additional profits (ie, payoff) resulting from coalitions will be distributed by their equal participation role" Equal Sharing Role "to find optimal coalitions using the Pareto Order for power Transmission.
\end{abstract}

Keywords: Micro grids, Micro Station, (CG-TFS), Equal Sharing Role, Pareto Order.

\section{Introduction}

In recent years, power grids are experiencing a revolution-ray technological transformation. One significant characteristic is that electric appliances can receive realtime power price via communication networks and optimize its power consumption level according to current power price. Then, the utilization of power efficiency is consequently improved, and the global energy consumption minimized for enhancing the emergency of energy resource. In smart grid, a key exception is the manner of the communication network to deal with transfer rate obviously, the data flow of power price cannot be flexible because it should be with real time; otherwise, it may meet a substantial loss if the expired power price is used. Therefore, the data transmission of the energy price must be equipped with the mechanism of exchange guarantee [1]. So we study detailed mechanism of power price, and how to enable $\mathrm{MG}_{\mathrm{s}}$ to cooperate with each other's and obtain an optimal cost including various kind of expenditures and achieve the maximum profits produced from forming coalitions between micro grids or users by their "Shapley value",

* Corresponding author.

E- mail address: halagaber_1@yahoo.com 
Which provides provably-good performance algorithm needed for the enhance routing in smart grid [2]. First, we study power transaction model comprising a various of information, such as electricity generation of various energy resources, user demand, topological structure of MGs network and different kinds of disbursement. In this paper, we study how to enable MGs cooperate with each other's to obtain an optimal cost including various kind of disbursement. This proposal based on Coalitional Game Theory. $\mathrm{MG}_{\mathrm{s}}$ first send their extra power and needs to MS. Subsequently, the MS performs mergeand-split operations to decide an optimal formation structure in turn of $\mathrm{MG}_{\mathrm{s}}$. After receiving formation direction from MS, then start power exchange with each other, which directly decrease their power transmission with MS. This inner power transfer will bring monetary benefit to $\mathrm{MG}_{\mathrm{s}}$ by saving cost of buying extra electricity and all improving power losses. In addition, power minimization approach across the entire smart grid and consider a fair payoff distribution for each member with the coalition by using Equal Sharing Role that reduce the repetition time to find optimal coalition.

The remainder of paper organized as follows. The background and related works discussed in Section II. The system model is discussed in Section III. In Section IV, simulation result and conclusion are indicated with Section V.

\section{Background and related works}

Communications infrastructure is required to communicate different devices on the smart grid. Integrated communications infrastructure must be implemented with the most priority for building smart grid efficiently. To find possible tears of the grid system, with high reliability, scalability, security, powerful and effectively cost communications infrastructure which supports requirements is certainly needed [3-5]. A smart MG network design proposed to satisfy demands of each MG with an optimized buying price. Aforementioned works mainly interested by the optimization of one individual aspect (e.g. buying price or power loss), our paper aims to provide an exhaustive financial power transaction to optimize financial efficiency of all MGs and performance of network enhancement. In this regard, there are many factors that should be combined. For instance, transmission cost is often required in the real electricity market to control the transmission congestion [6]. All $\mathrm{MG}_{\mathrm{s}}$ are linked to the MS by common distribution lines, through which they can perform power transfer with MS and other $\mathrm{MG}_{\mathrm{s}}$ when there are power surplus or shortage. Because of the higher expenses (e.g. buying price and power loss) of the power transfer among $\mathrm{MG}_{\mathrm{s}}$ and the $\mathrm{MS}, \mathrm{MG}_{\mathrm{s}}$ are [7] expected to exchange complementary power with each other's Finally, the Coalition Game Theoretic Formulation Strategy for the $\mathrm{MG}_{\mathrm{s}}$ applied to multiple $\mathrm{MG}_{\mathrm{s}}$ scenarios and therefore enhances the flexibility and capability of $\mathrm{MG}_{\mathrm{s}}$ network and will distribute all extra profits that improve the system performance. At present, coalition game theory applied to cooperation-based problems in smart grid, which shows a great performance in scalability and flexibility [1,8-11]. Saad et al. [8] presented a novel cooperative strategy based on theory of coalitional game that $\mathrm{MG}_{\mathrm{s}}$ can form coalitions and coordinate power bargains within coalitions to minimize the power losses over distribution lines. Wei et al. [9] suggested a greedy coalition algorithm to reduce the power loss of $\mathrm{MG}_{\mathrm{s}}$ with the consideration of energy storage. While the aforesaid works mainly focus on the optimization of one individual mien (e.g. purchase cost or power loss), our paper aims to provide a cooperative power transaction model to optimize Frugality efficiency of the whole $\mathrm{MG}_{\mathrm{s}}$ network. In this regard, many factors should be combined and analyzed. As illustrate by example, transmission cost is often required in the real electricity 
market to control the transmission restriction. Besides, the compensation cost (load shedding or load profit), accompanying with compensation costs, has shown to be an efficient method to overcoming the energy shortages of $\mathrm{MG}_{\mathrm{s}}$. we can apply these factors to multiple $\mathrm{MG}_{\mathrm{s}}$ Schemes for improving the flexibility and capability of $\mathrm{MG}_{\mathrm{s}}$ network, so few of the existing works have combined these factors into their energy trading framework.

\section{System model}

In this section, we describe the way of power transaction among MGs and MS by Conventional Model. The corresponding system model is shown in Fig. 1 [14].

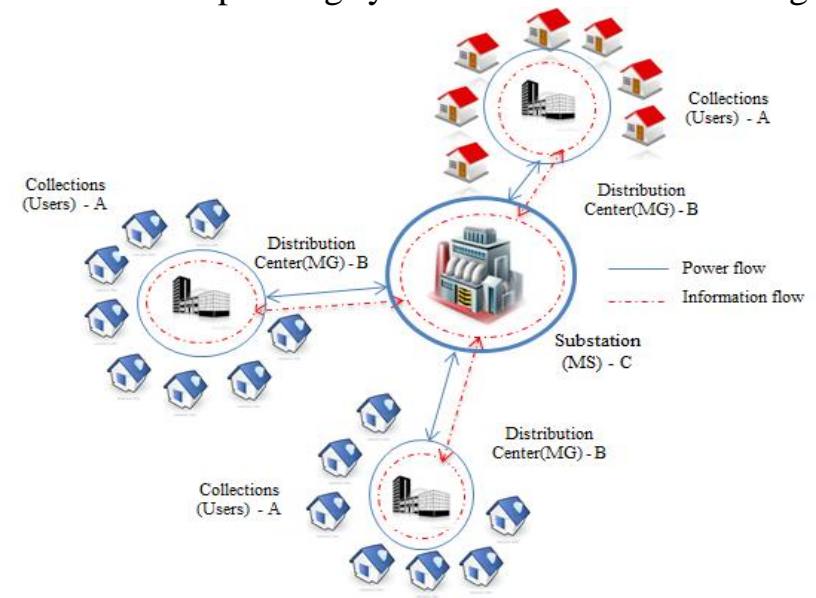

Fig. 1. Conventional Model for multiple MGs network

\subsection{Conventional model}

The Conventional system is placed at three levels with their functionality, they are (Collections (users), Distribution Center $\left(\mathrm{MG}_{\mathrm{s}}\right)$ and Substation) respectively. The functionality of each one will describe below. Collections (users) are sets of lowest level of agents that are purely belonging to a single smart home or they can be embedded into smart meter. The overall responsibility of collections is to minimize the cost of electricity for a single smart home which is connected. To achieve these objective collections will predict the load of electricity one-hour prior use. It will also maintain a flexible and inflexible load record to estimate the elasticity of the expected load change. It will also calculate electricity cost to get required power $(\mathrm{Q})$ from micro-grids. Some users combined together forming collection. According to proposed system model a fixed amount of smart homes are grouped to form collections that managed by Distribution Center $\left(\mathrm{MG}_{\mathrm{s}}\right)$ as shown in figure 1[14]. The main task of Distribution Center (MGs) is calculating the cost to get required power (Q) of smart home from its MGs if its amount of electricity demanded cannot be fulfilled, they sent Acknowledgement to distribution center and Substation or micro station (MS) by the required power with comparing the cost, to get the least one in cost and achieving its demanded. The third and supervisory group of $\mathrm{MG}_{\mathrm{s}}$ is called Substation that deals directly with main power grid. Substation will calculate expenditure of required power $(\mathrm{Q})$ to get from main power grid if it cannot be fulfilled from both micro grids and Collections (users), finally the flowchart that describe the conventional system as per indicated Fig. 2. 
In conventional model, $\mathrm{MG}_{\mathrm{S}}$ works individually and only performs energy trading towards a macro station (MS), which is the primary substation connected to utility grid, so this system consider non-cooperative model, where each MG only exchange power with the MS.

Because in non-cooperative case each MG can be regarded as a coalition, the payoff of MG is equal to that of coalition. Thus, we able to define the non-cooperative payoff (utility) of each $\mathrm{MG}_{\mathrm{i}}$ as the total power loss because the power transfer besides exchanging power with the MS, the MGs can exchange power with others. Because power loss during transmission among the neighbouring MGs are always less than that between the MS and a MG, the MGs can form cooperative groups, referred to coalitions throughout this paper, to exchange power with others, to minimize the power loss in the main smart grid and maximize their payoffs.

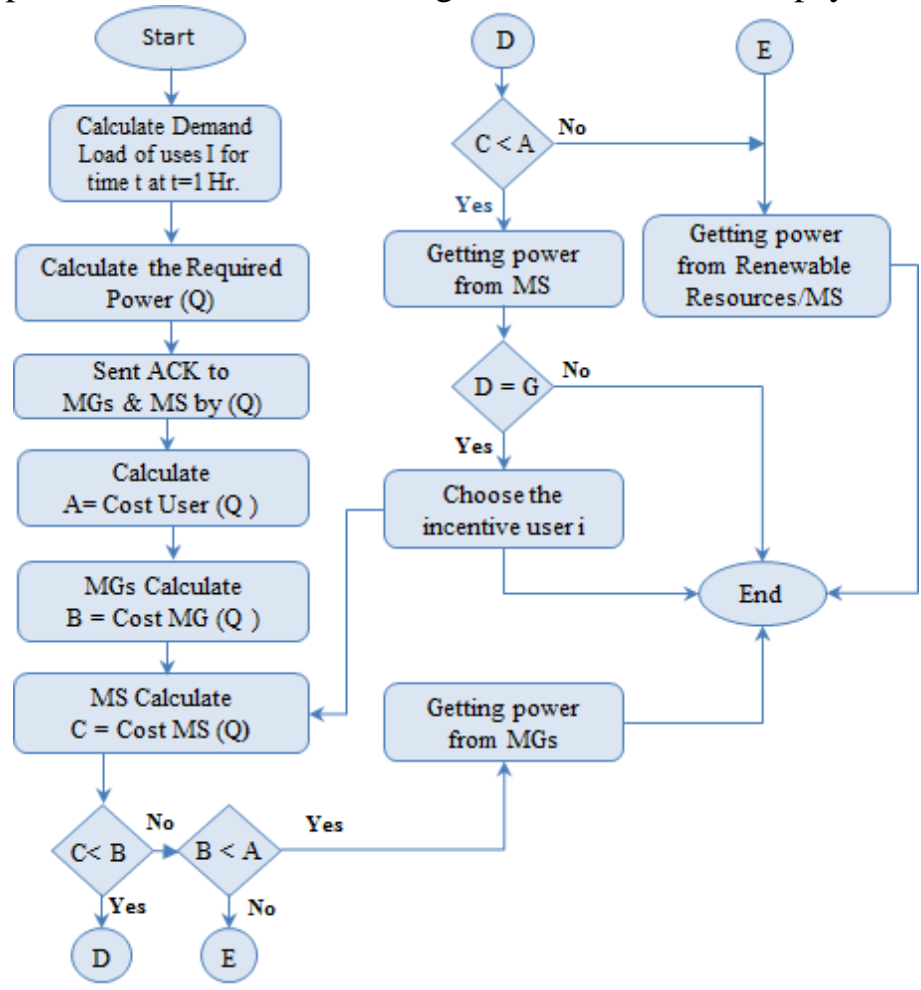

Fig. 2. Conventional Model Flow Chart

Most important factors that can be used for improving the performance of $\mathrm{MG}_{\mathrm{s}}$, is how to achieve Maximum payoff between $\mathrm{MG}_{\mathrm{s}}$ and Users due to coalitions. When MG's electricity generation is insufficient or excessive, it needs to export extra power to or import additional power from MS. With advent of $\mathrm{MG}_{\mathrm{s}}, \mathrm{MG}_{\mathrm{s}}$ can form networks and exchange information with each other's. it is possible for $\mathrm{MG}_{\mathrm{s}}$ to autonomously perform energy management and make decisions on cooperative transaction. In this context, there has been rising trend to study the cooperative mode of $\mathrm{MG}_{\mathrm{s}}$. MS, which works as a typical wholesaler in the power market [1], takes in charge for group of $\mathrm{MG}_{\mathrm{s}}$ and performs central control of the coordination of $\mathrm{MG}_{\mathrm{s}}$. Utility grid usually has different sell rate and buy rate [2] when trading with MS. For instance, when $\mathrm{MG}_{\mathrm{s}}$ have power sell and transfer to MS, utility grid will sell the extra electricity from MS at the rate $S_{\text {Rate }}$. On other hand, when MGs meet with power shortage,

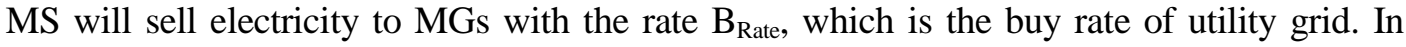
most cases, $B_{\text {Rate }}>S_{\text {Rate }}$ which ensures the profit of the utility grid. There exist drawbacks in 
the conventional mode that it will increase cost of $\mathrm{MG}_{\mathrm{s}}$ as keep MS with a high level of power load. Furthermore, $\mathrm{MG}_{\mathrm{s}}$ are usually located near to groups of end users (EUs) in different areas. The distributive deployment will give rise to a long distance between $\mathrm{MG}_{\mathrm{s}}$ and MS, which makes power losses more noticeable and large. Therefore, the $\mathrm{MG}_{\mathrm{s}}$ have an incentive to work in a cooperative way to decrease the unnecessary power transmission between MG and MS. To improve economic efficiency of the cooperative mode, there is a need to study the power transaction Model comprising several of information, such as electricity generation of various energy resources, user demand, topological structure of MGs network and different kinds of expenses. As different cooperative pairs of MGs lead to different costs, so we indicate how to determine criteria required for making the remains of MGs to collaborate to each other's to improve their efficiency.

\subsection{Cooperative coalition model}

According to Fig.3, Each MG can perform sole energy management. Each MG can perform information exchange with other MGs and the MS through the communication network. Besides, the MG can receive formation instruction.

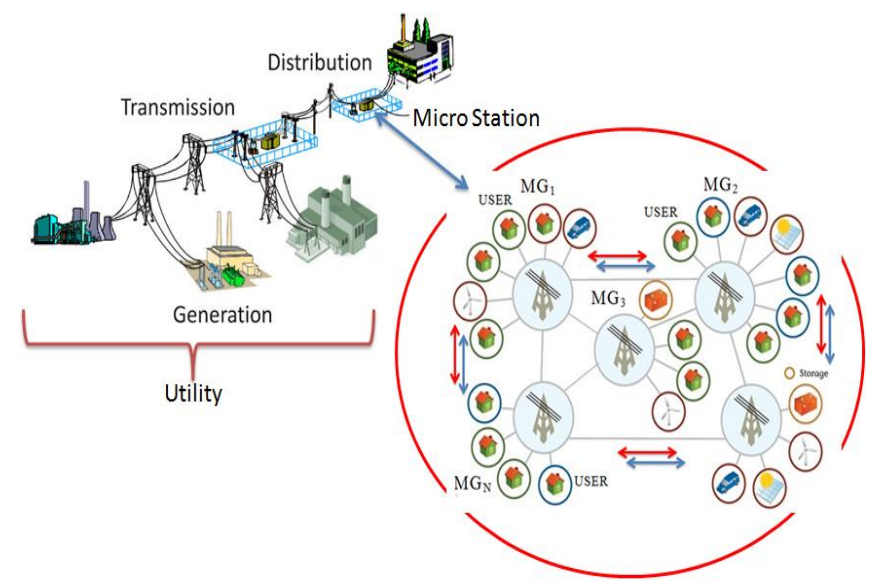

Fig. 3. Cooperative Model for multiple MGs network with Coalitions

Other $\mathrm{MG}_{\mathrm{s}}$. Taking $\left\{\mathrm{MG}_{1}, \mathrm{MG}_{2}, \mathrm{MG}_{3}\right\}$ as example, each one of these three $\mathrm{MG}_{\mathrm{s}}$ can send their information (e.g. extra power or demand, location) to the MS. Conversely, there they can receive coalition formation information from the MS, while MG2 has additional power to sell and $\mathrm{MG}_{1}$ and $\mathrm{MG}_{3}$ require power that available at $\mathrm{MG}_{2}$ Subsequently, $\mathrm{MG}_{\mathrm{s}}$ receive the coalition formation instruction and the agents start to establish power transfer connection. Finally, local transfer starts and $\mathrm{MG}_{2}$ sells excessive electricity to $\mathrm{MG}_{1}$ and $\mathrm{MG}_{3}$ by forming a coalition. Let $\mathrm{N}$ denotes the set of all $\mathrm{MG}_{\mathrm{s}}$. Assume that one day divided into 24 periods, each representing $1 \mathrm{~h}$. In each period, the power generation of $\mathrm{MG}_{\mathrm{i}}$ is $G_{i}$ and the total demand of its $\mathrm{EU}_{\mathrm{s}}$ is $D_{i}$, wher $G_{i}$ and $D_{i}$ are both active powers. We noted that reactive power for loads is assumed sufficient during all periods in our model as Fig.3 depicts. Taking MG and its users as a whole, the real quantity

$r e q_{i}=G_{i}-D_{i}$ defined as the total extra or required (demand) of $\mathrm{MG}_{\mathrm{i}}$. Additionally, $\theta_{\max }$ is supposed to be maximum capacity percentage of the allowable loads that can be cut off. Therefore, the real function is $r e q_{i}=G_{i}-\left(1-\theta_{\max }\right) D_{i}$ when considering load profit. For each $\mathrm{MG}_{\mathrm{i}}, r e q_{i}>0$ means that it generates excessive electricity and can sell it to others; $r e q_{i}<0$ implies that its demand cannot be satisfied and wants to buy electricity from others; $r e q_{i}=0$ 
represents that its production balances its demand. The MGs with $r e q_{i}>0$ constitute the set of sellers $S_{s}$ while the $\mathrm{MG}_{\mathrm{s}}$ with $r e q_{i}<0$ forming the set of buyers $S_{b}$. According to [12], the generation $G_{i}$ and demand $D_{i}$ are usually considered as random numbers. To achieve a more cost-efficient power transaction, the $\mathrm{MG}_{\mathrm{s}}$ are willing to directly trading energies between sellers and buyers when it gains benefits than trading with the MS.

Our Model considers several of expenditures. For each $\mathrm{MG}_{\mathrm{i}}, C_{g}$ represents the electricity generation cost, which depends on

$Q=\left\{\begin{array}{cl}Q_{b u y} & =r e q_{i}>0 \\ Q_{\text {sell }} & =r e q_{i}<0 \\ 0 & =r e q_{i}=0\end{array}\right\}$

The dominant renewable source type. For simplicity, $C_{g}$ is assumed to be same for all MGs. $C_{b}$ is the Buying rate for $\mathrm{MG}_{\mathrm{s}}$ to buy electricity from the MS. It fluctuates with various periods of time and it will be higher in peak time. $C_{l}$ is the transmission cost per unit distance. As mentioned before, the $\mathrm{MG}_{\mathrm{s}}$ are supposed to transfer energy on the common distribution line. Intuitively, the longer the power line between two trading $\mathrm{MG}_{\mathrm{s}}$ is, the more influence (e.g. the risk of congestion) they will bring to distribution network. Naturally, the transmission cost is assumed to be with respect to distance. $C^{c o m}{ }_{l}$ denotes the communication cost per unit distance. $\mathrm{MG}_{\mathrm{s}}$ exchange information through the communication network that requires certain expenditure. Similar to transmission cost, the communication cost considered as a function of connection distance. $S_{\text {shed }}$ denotes the compensation cost paid to the cut off loads after perform load shedding. In real case, $S_{\text {shed }}$ will be different according to necessity of the interruptible loads. In [1, 8], Coalitional Game Theory applied in smart grids to minimise power losses by forming cooperative groups. Basically, a coalitional game defined as a three tuple $(\mathrm{N}, \mathrm{n}, \varphi)$ [13], where $\mathrm{N}=\{1,2, \ldots, \mathrm{N}\}$ is sets of players, $\mathrm{n}: 2^{\mathrm{N}} \rightarrow \mathrm{R}$ is utility function corresponding for each coalition $S \subseteq \mathrm{N}$ that defines the overall payoff achieved by $S$ and $\varphi$ is a vector which represents the obtained payoffs of each member. In this paper, each MG is taken as a player, which finds other $\mathrm{MG}_{\mathrm{s}}$ for forming a coalition to achieve economic savings for itself and the whole coalition. Each coalition divided into two subsets: the sellers' set $S_{s}$ and the buyers' set $S_{b}$. In each coalition, the 'seller' MG will directly trade electricity with 'buyer' MG. After the local power transfer is done, the coalition will perform transaction with MS if there is still extra power or demand. Obviously, it should be at least one seller and one buyer in any coalition $S$. As basis of analysing cooperative behaviours of the players, the payoff function is first defined here. In this problem, our aim is to minimise the total power transaction cost of $\mathrm{MG}_{\mathrm{s}}$. For each coalition $S$, the overall cost in a given time period $\mathrm{t}$, consist of four parts:

- The power buying rate for each 'buyer' MG to buy electricity from other $\mathrm{MG}_{\mathrm{s}}$ or the MS.

- The cost spent on power losses rate value.

- The communication rate value for each MG to exchange information with other MGs.

- The compensation rate for the load shedding or load profit.

In our model, the "seller" teams are supposed for selling electricity to others at a cost of generating cost. We noted MGs 'seller' have a profit from reducing the total charge distribution.

As a result, the profit of selling is not considered here. Hence, the overall payoff function of a coalition $S$ as 


$$
u(S . \Omega)=-\left(\sum_{i \in S b} V_{i}^{D}+\sum_{i \in S} V_{i}^{L}+\sum_{i \in S} V_{i}^{C}+\sum_{i \in S} V_{i}^{S}\right)
$$

Where $\Omega$ is the joining order of the buyers in the coalition $S$. Besides, the minus here is to convert the minimisation into the maximisation problem. For each buyer $\mathrm{MG}_{\mathrm{i}}, V_{i}^{D}$ represents + cost of buying electricity from $\mathrm{MG}_{\mathrm{s}}$ and MS. When buying from any other $\mathrm{MG}_{\mathrm{j}}$, the cost $C_{i j}$ comprises two parts: the generation cost $\mathrm{Cg}$ and the transmission $\operatorname{cost} C_{l}$, which can be denoted as

$$
C_{i j}=C_{g}+l_{i j} C_{1}
$$

this problem used to determine the maximum overall payoff of a coalition. Note that, the overall payoff based on the power transaction between $\mathrm{MG}_{\mathrm{s}}$ and $\mathrm{MS}$. by other words, the matching pairs of seller $\mathrm{MG}_{\mathrm{s}}$ and buyer $\mathrm{MG}_{\mathrm{s}}$ be difference in the total payoff. According to (3), the sell rate (sell cost) $C_{i j}$ within each two $\mathrm{MG}_{\mathrm{s}}$ is comprised of generation cost $\mathrm{C}_{\mathrm{g}}$ and transmission cost $C_{l}$. In our Model, we assume the buy rate (buy cost) $C_{b}$ is much larger than the generation cost $C_{g}$. Moreover, the transmission cost is assumed to be proportionate with the distance between MGs. For those MGs which are close to each other, the transmission cost $C_{l}$ is relatively small. Therefore, if those $\mathrm{MG}_{\mathrm{s}}$ near each other's form coalitions and perform local power transfer within the coalition, the total buying rate tends to be lower than buying from MS.

$$
V_{i}^{D}=\sum_{j \in S s} C_{i j} P_{i j}+C_{b} P_{i o}
$$

Here $P_{i j}$ is the power transmitted between these two $\mathrm{MG}_{\mathrm{s}}$ and $P_{i o}$ is the power transaction with $\mathrm{MG}_{\mathrm{i}}$ and $\mathrm{MS}$ if there remains power extra or required in $\mathrm{MG}_{\mathrm{i}}$. Before we design the payoff function of coalition, let us see some parameters. In this case, each MG exchanges power with the MS. In general, the medium voltage of power transfer between MG and MS is $U_{0}$. Any power transfer between MG and MS is accompanied with power loss. In process of power transfer, we only consider two kinds of power loss, namely (i) the power loss over the distribution lines inside the network, and (ii) the power loss due to other factors in the MS such as voltage conversion, dust, and so forth. we able to defined the power loss $V_{i o}{ }^{L}$ as follows.

$$
V_{i o}^{L}=\left(\begin{array}{ll}
R_{i o} & P_{i o}^{2} / U_{o}{ }^{2}
\end{array}\right)+\alpha P_{i o}
$$

Where $R_{i o}$ is the distribution line resistance between $\mathrm{MS}$ and $\mathrm{MG}_{\mathrm{i}}$, and $\alpha$ is a fraction of power loss caused by other factors. For simplicity, $\alpha$ is considered as a constant.

In a formed coalition, there many $\mathrm{MG}_{\mathrm{s}}$, which are to exchange power with others or even with the MS. Let a "seller" and a "buyer" be denoted by $\mathrm{MG}_{\mathrm{i}} \in \mathrm{S}_{\mathrm{s}}$ and $\mathrm{MG}_{\mathrm{j}} \in \mathrm{S}_{\mathrm{b}}$, respectively. If $\mathrm{MGi}$ and $\mathrm{MG}_{\mathrm{j}}$ want to exchange power, power loss function $V_{i j}{ }^{L}$ can be expressed as follows.

$$
V_{i j}^{L}=R_{i j} P_{i j}^{2} / U_{1}^{2}
$$

Where $R_{i j}$ is the resistance of the distribution line between $\mathrm{MG}_{\mathrm{i}}$ and $\mathrm{MG}_{\mathrm{j}} . U_{l}$ denotes the transfer voltage between $\mathrm{MG}_{\mathrm{i}}$ and $\mathrm{MG}_{\mathrm{j}}$ and it is lower than $U_{0}$. Because there is no voltage conversion between two $\mathrm{MG}_{\mathrm{s}}$, when power is transmitting among $\mathrm{MG}_{\mathrm{s}}$, we only calculate the transfer power loss among MGs. by other words, eq. (6) is a special case of eq. (5), when $\alpha=0$. After the local power transfer. To minimise the sell cost, $\mathrm{MG}_{\mathrm{s}}$ with closest distance predicted to form pairs to transfer power. Similarly, from (7) 


$$
V_{i}^{L}=\sum_{j \in S} C_{i j} V_{i j}^{L}+C_{b} V_{i o}^{L}
$$

Is the communication cost for $\mathrm{MG}_{\mathrm{i}}$ to exchange information with other $\mathrm{MG}_{\mathrm{s}}$ with same coalition via the communication network. As the communication cost per unit distance is represented as $C^{\mathrm{com}}{ }_{l,} V_{i}^{C}$ is calculated as in (8).

$$
V_{i}^{C}=\sum_{j \in S\{i\}} L_{i j} C_{1}^{c o m}
$$

The compensation cost or load profit is determined by the demand of each $\mathrm{MG}_{\mathrm{i}}$ is the expenditure for $\mathrm{MG}_{\mathrm{i}}$ to compensate for the loads that are cut off. As the total capacity of interruptible loads is $\theta_{\max } D_{i}, V_{i}^{S}$ can be denoted

$$
V_{i}^{S}=C_{i j} \theta_{\max } D_{i}
$$

For a coalition $S$, assume that its buyer subset $S_{b}$, has k buyers and the buyers join the coalition in the order $\Omega \in \Omega_{\mathrm{S}}$, which can be denoted as $S_{b}=\left\{\mathrm{b}_{1}, \ldots, \mathrm{b}_{\mathrm{k}}\right\}$. Each buyer $\mathrm{b}_{\mathrm{i}}$ is considered sequentially with the given order. For each $\mathrm{b}_{\mathrm{i}}$, the sellers $S_{j} \in S_{s}$ are sorted with their distance with $b_{\mathrm{i}}$ then buyer $\mathrm{b}_{\mathrm{i}}$ tries to buy from the first $S_{j}$ in $S_{s}$ If the residual power surplus of $S_{j}$ can satisfy the need of $\mathrm{b}_{\mathrm{i}}$ including the power losses as eq. (10)

$$
P_{s j} \geq-Q_{s j}+\frac{R_{i j}\left(-Q_{s j}\right)^{2}}{U_{0}{ }^{2}}
$$

Then the buyer $b_{i}$ does not require further transaction and the next buyer starts acting. Otherwise, $b_{i}$ buys all the possible power $s_{j}$ can provide, and then requires power transaction with the next closest seller $\mathrm{sj}+1$. The process is repeated until all buyers in Sb have no power demand, or none of the sellers in $S$ s have energy to trade. Then, the coalition $S$ will transmit power with MS if there remains power surplus or demand. By performing this matching process, the maximum utility function satisfying (11) of coalition $S$ obtained.

$$
\boldsymbol{U}(S)=\operatorname{Max}_{\Omega \in \Omega S} u(S . \Omega)
$$

This represents the maximum total utility produced by any $S \subseteq \mathrm{N}$. This represents that the minimum power loss over the distribution lines. Therefore, comparing with the noncooperative case, total summation of utilities of $\mathrm{MG}_{\mathrm{s}}$ in considered coalitions increase. That is to say, the $\mathrm{MG}_{\mathrm{s}}$ produce the extra profits through forming the coalition. Upon completion of coalition formation, the $\mathrm{MG}_{\mathrm{s}}$ belonging with same coalition face the problem of how to distribute all extra profits appropriately in the coalition. If the allocation of profits is not appropriate, the coalition will be split into parts. Thus, we need an appropriate allocation for the profits. For this purpose, we choose the "Equal sharing" role concept from cooperative game theory [14]. In each the cooperative game a unique sets distribution (between players) of the total product surplus by coalition of all the players. When a MG joins in a coalition, it will bring income for coalition. However, different order that MGs joined with coalition means different income. As we know traditionally, Shapley value [15] would be employed, but computing Shapley value requires iterating over every partition of a coalition, an exponential time endeavor. Another rule for payoff division is equal sharing of the profit among members. Equal sharing provides a tractable way for determine the shares and successfully used as an allocation rule in other systems where tractability is critical (e.g., [15]). For this reason, we adopt here the equal sharing of the profit as the payoff division rule 


$$
\varphi_{i(U)}=\frac{U(S)}{|S|}
$$

The formula interpreted as follows. Assuming that the coalition consists of one player at a time, each player asks for his contribution as an appropriate compensation, then mediates more than the various potential differences that can form a coalition. The function $U$ of MG is given. Hence, the "Equal sharing" value can be calculated. Furthermore, since the payoffs depend on MGs' order in the coalition, payoffs are likely to be different in different orders. In fact, the contribution of a player to the coalition depends on the order. Therefore, the fraction in eq. (12) attempts to calculate the average of payoffs in all conditions, and this average is the contribution of the player to the coalition [16]. A solution concept for coalitional games as a payoff vector which allocated each payoff among the players in fair manner. The primary concern for any coalition game is the stability. One of concepts solution concepts used to assess the stability of coalitions is the core. To maximize eq. (11), a strategy will be designed that can find the coalition having the $\mathrm{MG}_{\mathrm{s}}$ have certain minimum power loss with the coalition and the MS, or obtain maximum profit from forming the coalition. for achieving this target, $\mathrm{MG}_{\mathrm{s}}$ calculate the value of Difference of Power loss per unit Power (DPUP) between within coalition and out of the coalition. Obviously, the greater difference will bring greater payoff for coalition. If $\mathrm{MG}_{\mathrm{i}}$ wants to form coalition with $\mathrm{MG}_{\mathrm{j}}$ and the quantity of transfer is $\mathrm{Q}$, the function of DPUP between them is as follow.

$$
\operatorname{DPUP}_{(\mathrm{i} . \mathrm{j})}=\frac{\mathrm{V}_{\mathrm{io}}^{\mathrm{L}} Q_{\mathrm{ij}}+\mathrm{V}_{\mathrm{jo}}^{\mathrm{L}} Q_{\mathrm{ij}}+\mathrm{V}_{\mathrm{ij}}^{\mathrm{L}} Q_{\mathrm{ij}}}{Q_{\mathrm{ij}}}
$$

Where $V_{i o}{ }^{L}, V_{l o}{ }^{L}$ and $V_{i j}{ }^{L}$ are given by eqs. (5) and (6). In other words, the function of DPUP is the marginal value of a $\mathrm{MG}$ for coalition. For maximizing the profit of coalition, MGs will find the partners are able to maximize the eq. (11). From eq. (12), we see that if two MGs have equal contributions to the coalition, their corresponding Shapley values are the same, although their individual values are different. Furthermore, the value depends on the order of $\mathrm{MG}$ in the coalition. When the result appears to be unfair; however, it indicates the practical contribution of players to the coalition. Therefore, $\mathrm{MG}_{\mathrm{s}}$ with same coalition distribute the extra payoff, based on eq. (12). Based on an envisioned strategy for objective selection and concept of Shapely value for extra profit distribution in a coalition, we suggest an algorithm to formulate distributed coalitions of MGs in remainder of this section. First, we introduce an important definition from.

Consider two disjoint coalitions $A=\left\{A_{1}, \ldots, A_{i}\right\}$ and $B=\left\{B_{1}, \ldots, B_{j}\right\}$ which are formed out of the same players. For one coalition $A=\left\{A_{l}, \ldots, A_{i}\right\}$, the payoff of player $\mathrm{k}$ in coalition $A_{k}$ $\in A$ is $\Phi_{k}(A)=\Phi_{k}\left(A_{k}\right)$ where $\Phi_{k}\left(A_{k}\right)$ is given by (11) for coalition Ak. Coalition $A$ is preferred over $B$ by Pareto order

$$
A \triangleright B \Leftrightarrow \Phi_{j}(A) \geq \Phi_{j}(B) . \quad \forall j \in A . B
$$

And $\quad \Phi_{k}(A)>\Phi_{k}(B) . \quad \exists k \in A . B$

With at least one severe inequality ( $>$ ) for a player k. Pareto's order means that a group of players would prefer to join $A$ rather than $B$, if at least one player can improve his profit. when the structure has been changed from $B$ to $A$ without cutting down the payoffs of any others.

When forming the optimal coalition structure, each MG prefers to attend in the coalition, which will bring most profits to it, while not the total coalition value. At the end, the comparison relation $[17,18]$ that is called 'Pareto order' based on an individual payoff 
is introduced. Consider two as (14) shows, coalition $A$ is a preferred structure than $B$ if at least one player able to gain a better payoff without reducing other players' payoffs. On basis of the individual comparison relation, we suggest an algorithm by the MS in a centralized way involving two rules:

Join and separate or (Merge and Split).

Merge: Merge any sets of coalitions $\left\{S_{l}, \ldots, S_{l}\right\}$ where

$\left\{\cup_{\mathrm{i}=1}^{\mathrm{L}} S_{i}\right\} \triangleright\left\{S_{l}, \ldots, S_{l}\right\}$, hence, $\left\{S_{l}, \ldots, S_{l}\right\} \rightarrow\left\{\cup_{\mathrm{i}=1}^{\mathrm{L}} S_{i}\right\}$

Split: Split any coalition $\left\{U^{\mathrm{L}}{ }_{\mathrm{i}=1} \mathrm{~S}_{\mathrm{i}}\right\}$ where

$$
\left\{\left\{S_{l}, \ldots, S_{l}\right\} \triangleright\left\{\cup_{\mathrm{i}=1}^{\mathrm{L}} S i\right\} \text { hence, }\left\{\cup_{\mathrm{i}=1}^{\mathrm{L}} S_{i}\right\} \rightarrow\left\{S_{l}, \ldots, S_{l}\right\} .\right.
$$

From the definitions of merge and split, we find that some $\mathrm{MG}_{\mathrm{s}}$ and some $\mathrm{MG}_{\mathrm{s}}$ coalitions will have joined with a new coalition or merge with a grand coalition, respectively, at least one of them can improve its payoff and do not cut down the payoffs of any other $\mathrm{MG}_{\mathrm{s}}$ and coalitions, respectively. On other hand, a big coalition will be split into some small coalitions (or even disappear) if the $\mathrm{MG}_{\mathrm{s}}$ find that they can leave the coalition or merge with other coalition, so as to get more payoffs than that in the current coalition. Hence, a merge or split decision by Pareto order will ensure that all the involved MGs agree on it. Applying the merge and split rules, coalitions will change their structure and yield better individual payoffs of each MG, thus improving the whole profits. In [1, 17-18], the convergence of the merge-and-split operation has been proved. The detail as in Fig. 4. we noted that merge and split are rules for determining the other stages: request exchange, Merge-and-split and cooperative transaction. Figs 4 show the flow chart of the total system.

\section{Simulation result}

In this section, simulations experiments are performed to validate our proposed model. For the simulation, a distribution network of an area $100 \times 100 \mathrm{~km} 2$ is set up. As Fig. 3 shows, MS is located at the centre of upper border and the $\mathrm{MG}_{\mathrm{s}}$ are deployed randomly in the network. Under the MS lies a common distribution power line, to which all $\mathrm{MG}_{\mathrm{s}}$ are connected in a radial pattern. In fact, our cooperative model applied to any other topological structures. Similarly, as [19], the power generation $G_{i}$ and demand $D_{i}$ of

$\mathrm{MG}_{\mathrm{i}}$ are assumed Gauss random variables distributed from 1 to $2.5 \mathrm{MW}$. Therefore, the power surplus or need $\mathrm{req}_{i}$ of $\mathrm{MG}_{\mathrm{i}}$ is a random variable distributed from -1.5 to $1.5 \mathrm{MW}$. we noted that our economic power transaction applied in real cases by changing the proportion of $\mathrm{MG}_{\mathrm{s}}$ with power surplus or power deficient in the distribution network.

The resistance on the distribution line of the network is set as $R=0.2 \Omega / \mathrm{km}$ with power transfer fraction of MS as $\alpha=0.02$ [18]. The medium voltage of the distribution line is set to $U_{0}=33 / 11 \mathrm{kV}$ [20]. The ampacity of the distribution network is assumed as $150 \mathrm{~A}$, which is typical value in distribution network. retail price for the MS and the generation cost of $\mathrm{MG}_{\mathrm{s}}$ are derived from the prices in California in 2018 [20]: $C_{b}=30$ cents $/ \mathrm{kW}$ and $C_{g}=10$ cents $/ \mathrm{kW}$, respectively. Here, we not consider different generation price $C_{g}$ for each MG with different energy mix to simplify the numerical simulation. The transmission cost is set to 0.03 cents $/ \mathrm{kW} \mathrm{km}$. The communication cost per unit distance is set to 0.3 cents $/ \mathrm{km}$. Compensation price for load shedding is set to 2.5 cents $/ \mathrm{kW}$ and the maximum capacity 
percentage of the interruptible loads $\theta_{\max }$ is set to $10 \%$. The simulation results are average values obtained after ten runs of the experiment with random deployment of the $\mathrm{MG}_{\mathrm{s}}$.

With increasing number of $\mathrm{MG}_{\mathrm{s}}$, the reduction rate also increases, as more $\mathrm{MG}_{\mathrm{s}}$ in distribution network implies a higher possibility to find MGs which have complementary surplus or demand and require lower purchase costs. Moreover, the cooperative model considering load shedding achieved lower total expenditure compared with the case not performing load interrupting when compensation cost is relatively low. In essence,

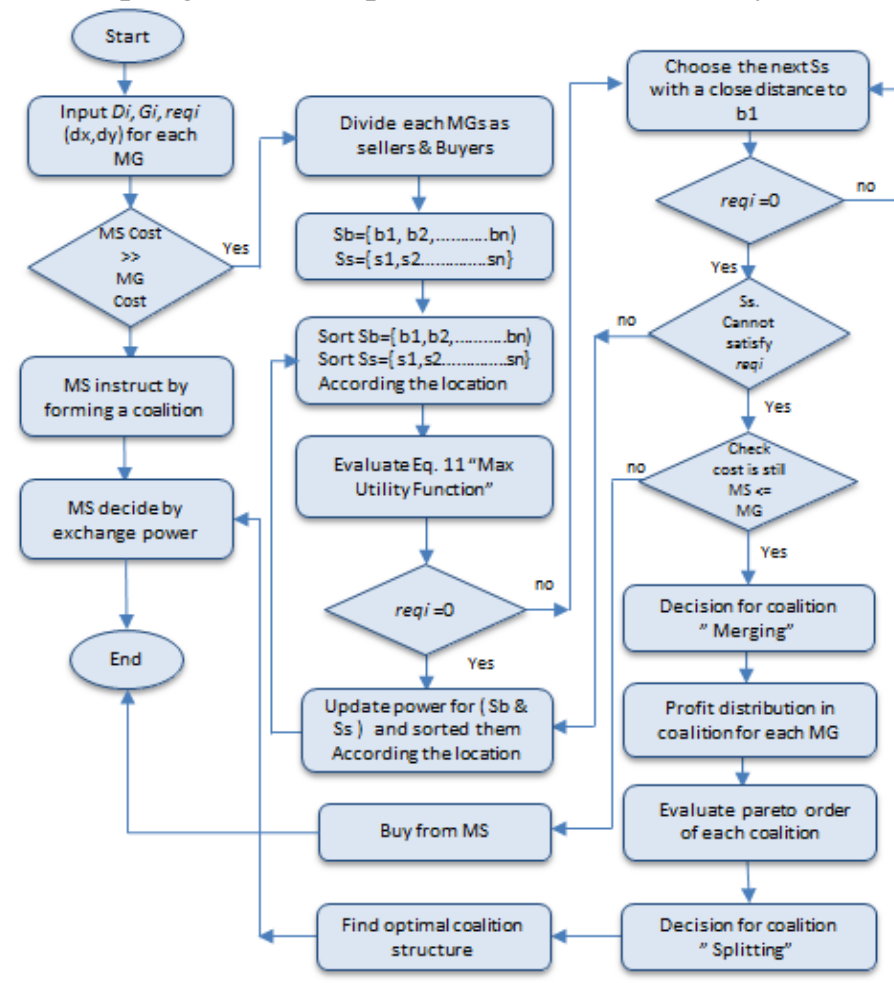

Fig. 4. Cooperative Model for multiple MGs network with Coalitions Flow chart

An interruptible load reduces the power need of $\mathrm{MG}_{\mathrm{s}}$ and hence decreases the power transaction between $\mathrm{MG}_{\mathrm{s}}$ and the MS.

Fig. 5 depicts the normalized total expenditure for all $\mathrm{MG}_{\mathrm{s}}$ managing their power surplus or need. Three models are considered here: non-cooperative model, proposed cooperative and cooperative without load shedding for a total number of $\mathrm{MG}_{\mathrm{s}}$ varying from 10 to 50. As indicated in the figure, our cooperative achieved a asignificant cost reduction compared with non-cooperative Model and the cooperative without considering load shedding. The resulting cost reduction is ascribed to the direct trading within $\mathrm{MG}_{\mathrm{s}}$, which requires less cost than the power transaction with MS. Furthermore, the losses of power are decreased by cooperative transaction since the distance within $\mathrm{MG}_{\mathrm{s}}$ are usually smaller than with MS. 


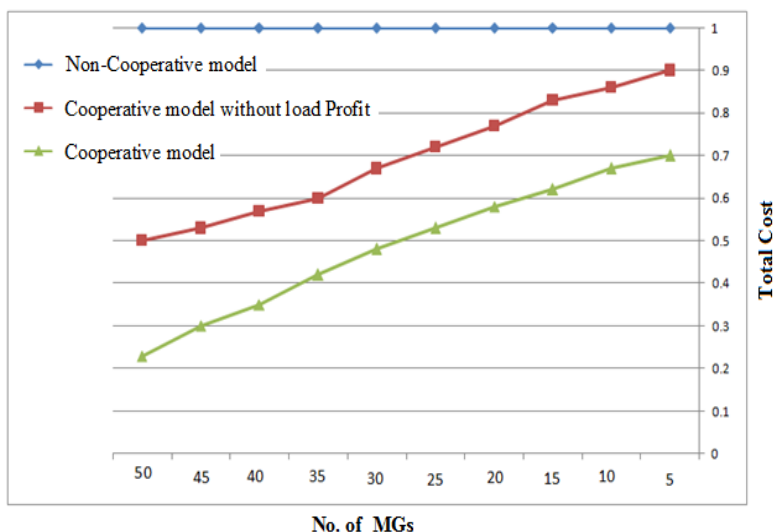

Fig. 5. Comparison of non-cooperative and cooperative models

Fig. 6 indicates how the transmission cost affects the cost reduction rate in the case when $\mathrm{N}=10$ and 50. With a higher transmission cost, the reduction rate decreases sharply and becomes near 0 . When the transmission cost gets higher, the expenditure for $\mathrm{MG}_{\mathrm{s}}$ to perform local transfer increases. This result indicates the significance of the pricing model of the utility grid. Introduced to facilitate direct trading within MG because it can effectively increase the utilization of the generated renewable energy and reduce the energy request from MS, which definitely decreases the generation of traditional plants.

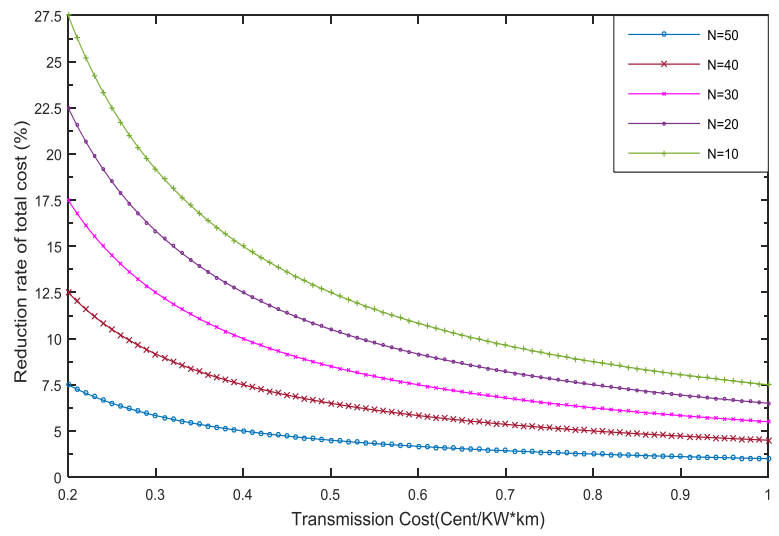

Fig. 6. Reduction rate of cost versus transmission cost

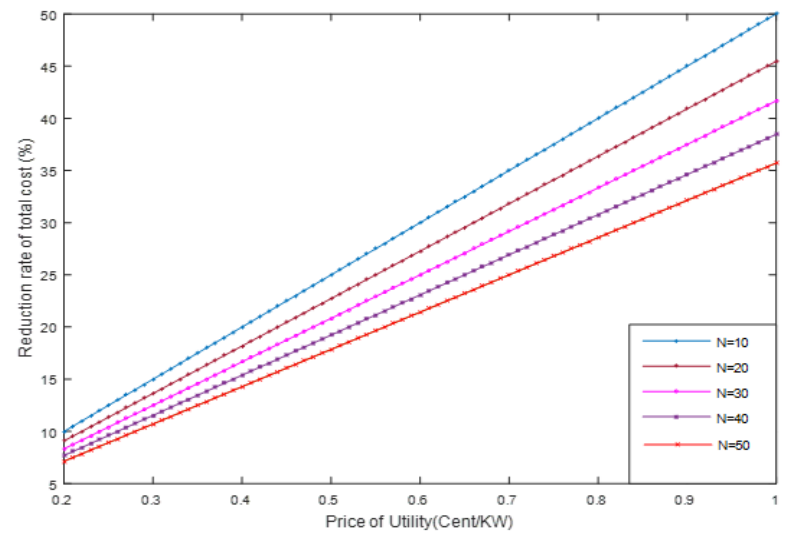

Fig. 7. Reduction rate of cost versus retail price 
Fig. 7 shows the relation between versus retail price of the utility grid and the cost reduction rate when the number of MGs is increased, it is shown that when the retail price is higher, the cost reduction rate becomes larger. Considering the price fluctuation during a whole day, since the electricity price of peak periods is larger than the peak-off periods, the MGs are more willing to form coalition to perform direct trading and thus gain more monetary profit in the peak periods than peak-off periods.

This after applied to the network shown in Fig. 6 Here, each MG will choose to trade with those MGs that have lower transmission costs subsequently while not considering other costs such as power loss cost and communication cost. our model can take a whole view of all MGs and obtain a more reasonable structure coalition, which makes each MG lower its cost. Table1. shows. The first column shows the id for each MG and the second column shows the deployment position of each MG. Columns 3, 4 and 5 show the total generation, total demand and power surplus or shortage of each MG, respectively. As it shows, we not assuming identical $\mathrm{MG}_{\mathrm{s}}$ at equal distances. each MG distance from MS and its position is determined randomly based on Gauss distribution, within a square of $100 \times 100 \mathrm{~km}^{2}$. The power surplus and shortage of each MG are considered Gauss random variables distributed from -2 to $2 \mathrm{MW}$. Fig. 8 gives the deployment of each MGs and MS based on data with Table 1.

Finally, the comparison results between our Cooperative and Non Cooperative Models to optimal payoff, when Applying merge and split rules, coalitions will change their structure and yield better individual payoffs of each MG, thus improving the whole profits.

Fig. 9 Show a comparison for two different methods, Equal Sharing Role and traditional method Shapley value for an appropriate allocation for the profits distribution, we noted that Equal Sharing Role is preferred to traditional method Shapley value due to decreasing the exponential time for iterating over every partition of a coalition specially when number of tasks increasing (no. of coalition) so the time factor to identify the best coalition required for power exchange, is more critical with smart grid network

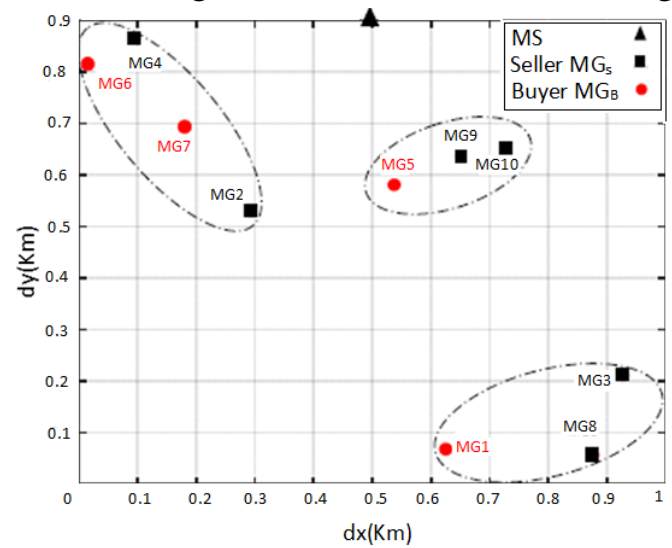

Fig. 8. Diffusion of $\mathrm{MG}_{\mathrm{s}}$ and $\mathrm{MS}(\mathrm{N}=10)$ 
Table 1.

Parameters of MGs in numerical experiments

\begin{tabular}{|c|c|c|c|c|}
\hline $\begin{array}{c}\text { MG } \\
\text { ID }\end{array}$ & $\begin{array}{c}\text { Deployment } \\
\text { Position } \\
(\mathrm{dx}, \mathrm{dy}) \mathrm{km}\end{array}$ & $\begin{array}{c}\text { Total } \\
\text { Generation } \\
\text { MW }\end{array}$ & $\begin{array}{c}\text { Total } \\
\text { Demand } \\
\text { MW }\end{array}$ & $\begin{array}{c}\text { Surplus/ } \\
\text { Shortage } \\
\text { MW }\end{array}$ \\
\hline 1 & $(0.6,0.09)$ & 1.815 & 2.420 & -0.605 \\
\hline 2 & $(0.3,0.0 .54)$ & 2.054 & 1.780 & 0.273 \\
\hline 3 & $(0.87,0.07)$ & 2.435 & 2.431 & 0.004 \\
\hline 4 & $(0.86,0.1)$ & 1.667 & 1.110 & 0.556 \\
\hline 5 & $(0.54,0.58)$ & 1.128 & 1.311 & -0.182 \\
\hline 6 & $(0.02,0.8 .1)$ & 1.086 & 2.163 & -1.077 \\
\hline 7 & $(0.19,0.69)$ & 1.944 & 2.371 & -0.427 \\
\hline 8 & $(0.87,0.16)$ & 2.194 & 2.174 & 0.020 \\
\hline 9 & $(0.65,0.63)$ & 2.037 & 1.443 & 0.593 \\
\hline 10 & $(0.73,0.65)$ & 1.518 & 1.228 & 0.290 \\
\hline
\end{tabular}

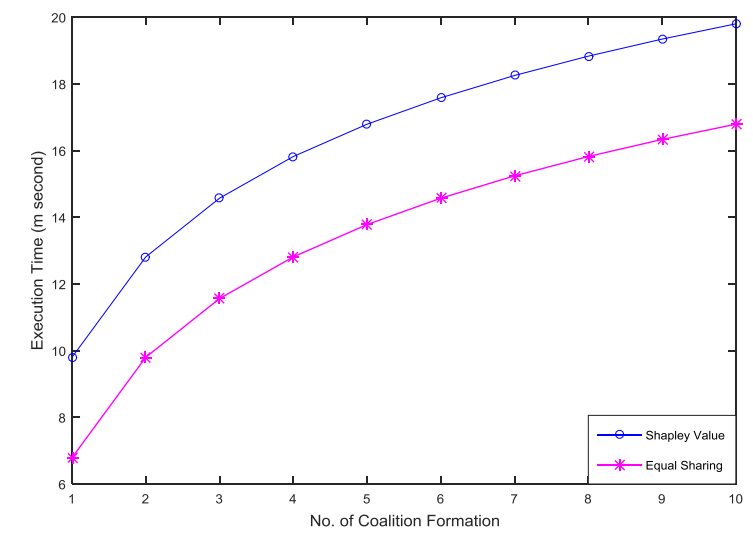

Fig. 9. Shapely Value and Equal Sharing Comparison

\section{Conclusion}

With this work, we suggest to how to obtain the best model for economic power coefficients in the MG network. It proposes a central algorithm mainly based on coalitional game theory that enable MGs to exchange the power between each other by forming coalitions, where they can perform direct energy trading satisfying their power surplus and demand. Since dealing directly with MGs avoids paying price differentials in the wholesaler, it requires a lower cost of trading with MS. In addition, MGs with short distances prefer to forming a coalition and thus reduce energy losses and other costs. Equal sharing role consider a tractable way, which is the easiest and fastest way to identify amount of participation any for coalition and was used successfully as a rule of allocation in other systems where traceability is very crucial to find the optimal alliance by distribute the profit with suitable manner.

Simulation results show the effectiveness of cooperative model over the noncooperative model, with significantly expenditure. Moreover, comparison results substantiate that compensation load improves the cost reduction rate. In future work, it will be interesting to study other successful roles of trading with more cooperative transaction and we want to incorporate the trust relationships among the MGs network. 


\section{REFERENCES}

[1] G. Xue, A. Sen, W. Zhang, J. Tang and K. Thulasiraman, "Finding apath subject to many additive QoS constraints," IEEE/ACM Transactions on Networking; vol. 15, pp. 201-211, 2007.

[2] NETL (National Energy Technology Laboratory), Appendix B1: A Systems View of the Modern Grid: Integrated Communications, Feb. 2007.

[3] G. Xue, W. Zhang, J. Tang, and K. Thulasiraman, "Polynomial time approximation algorithms for multi-constrained QoS routing," IEEE/ACMTransactions on Networking, vol. 16 , pp. 656-669, 2008.

[4] K. Moslehi and R. Kumar, "Smart grid - A reliability perspective," in Proc. of IEEE Innovative Smart Grid Technologies Conference (ISGT), 2010.

[5] Nenad A. Katic, Vlado Marijanovic, and Isabela Stefani, "Profitability of Smart Grid Solution application in distribution network", Power Generation, Transmission, Distribution and Energy Conversion (MedPower 2010), 7th Mediterranean Conference and Exhibition on, pp.1-6, 2010.

[6] Erol-Kantarci, M., Kantarci, B., Mouftah, H.T.: 'Cost-aware smart microgrid network design for a sustainable smart grid'. IEEE GLOBECOM Workshops, 2011, pp. 1178-1182.

[7] Ochoa, L.F., Harrison, G.P.:'Minimizing energy losses: optimal accommodation and smart operation of renewable distributed generation', IEEE Trans. Power Syst., 2011, 26, (1), pp. 198-205

[8] Saad, W., Han, Z., Poor, H.V.:'Coalitional game theory for cooperative micro-grid distribution networks'. IEEE Int. Conf. on Communications Workshops (ICC), 2011, pp. 1-5

[9] Wei, C., Fadlulah, Z.M., Kato, N.,et al.: 'On optimally reducing power loss in micro-grods with power storage devices', IEEE J. Sel. Areas Commun., 2014, 32, (7), pp. 1361-1370

[10] Ochoa, L.F., Harrison, G.P.:'Minimizing energy losses: optimal accommodation and smart operation of renewable distributed generation', IEEE Trans. Power Syst., 2011, 26, (1), pp. 198-205

[11] Deilami, S., Masoum, A.S., Moses, P.S.,et al.: 'Real-time coordination of plug-in electric vehicle charging in smart grids to minimize power losses and improve voltage profile', IEEE Trans. Smart Grid, 2011, 2, (3), pp. 456-467

[12] Li, H., Zhang, W.: 'Qos routing in smart grid'. Proc. of IEEE Global

Telecommunications Conf., Miami, FL, USA, 2010, pp. 1-6

[13] Lai, H.Q., Chen, Y., Liu, K.J.:'Energy efficient cooperative communications using coalition formation games', Comput. Netw., 2014, 58, pp. 228-238

[14] Y. Cheng, "Architecture and principles of smart grids for distributed power generation and demand side management," in SMARTGREENS, 2012, pp. 5-13.

[15] Lloyd. S. Shapley, "A Value for n-person Games", In Contributions to the Theory of Games, volume II, by H.W. Kuhn and A.W. Tucker, editors. Annals of Mathematical Studies v.28, pp.307-317. Princeton University Press, 1953

[16] O. Shehory and S. Kraus, "Task allocation via coalition formation among autonomous agents," in Proc. of Intl. Joint Conf. on Artificial Intelligence, vol. 14, 1995, pp. 655-661.

[17] H. Li, and W. Zhang, "QoS routing in smart grid", Proc. IEEE Global commun. conf., Miami, FL, USA, 2010

[18] Lai, H.Q., Chen, Y., Liu, K.J.: 'Energy efficient cooperative communications using coalition formation games', Comput. Netw., 2014, 58, pp. 228-238

[19] Apt, K.R., Witzel, A.: 'A generic approach to coalition formation'. Proc. of Int. Workshop COMSOC, Amsterdam, UK, 2006, pp. 1-21

[20] http://www.eia.gov/electricity/ January 4, 2018 


\section{توزيع الربح في شبكة الصغرى}

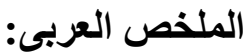

تعتبر الثبكات الذكية من أنظمة الطاقة المتطورة التي لديها القدرة على التحكم في تحميل الطاقة عن التعاء طريق توفير تكلفة شر اء الكهرباء الإضافية وتخفيف خسائر الطاقة. ويعتبر الإتصال بين الرئ المحطات الصنات الصغيرة بicro grids (MGs) بتكلفة الإرسال وتكلفة الطاقة المتبادلة التي تمثل دورًا فعالاً في إستقرار الأسعار للشبكات الذيكة الذية الصغيرة . لذلك ، نقترح آلية التبادل في المحطات الصغيرة ( MGs ) للتكامل ، وتحقيق جميع المتطلبات الخاصة

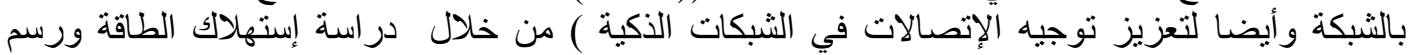

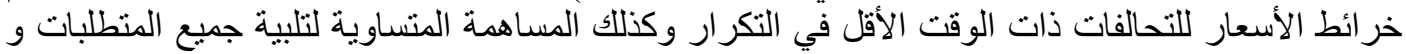

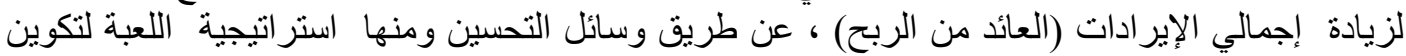

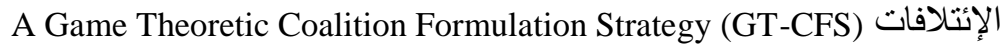
و التي تستخدم لسر عة التوجيه العالية ولتلبية متطلبات الثبكة الذكية ، وسيتم توزيع الأرباح الإضافية (أي

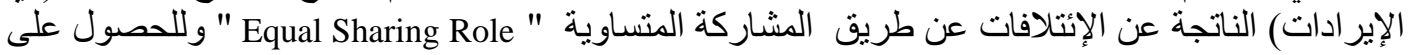
التحالفات المتلى لنقل الطاقة بإستخدم الإنغات عن طريق 